\title{
A165 ELEVATED EXPRESSION OF THE TH17 CYTOKINE INTERLEUKIN 22 IN RHEUMATOID ARTHRITIS
}

M Schramm, J Leipe, H Schulze-Koops, A Skapenko Division of Rheumatology, Medizinische Poliklinik, University of Munich, Germany

10.1136/ard.2010.129668b

Background and Objectives Interleukin 22 (IL22) is a pleiotropic cytokine with proinflammatory properties. It is primarily expressed by T helper 17 (Th17) cells and has recently been implicated in the pathogenesis of collagen-induced arthritis, a murine model of autoimmune polyarthritis. The authors were able to demonstrate a pathogenetically dominant role of Th17 cells and their signature cytokine IL17 in rheumatoid arthritis (RA) and psoriatic arthritis (PsA). Since the role of IL22 and its 
coordinate expression from Th17 cells in human autoimmune arthritis is unknown, our aim was to investigate IL22 in RA and PsA.

Methods and Results IL22 was analysed in well-defined cohorts of treatment-naive patients with active early RA and PsA. As control cohorts, the authors used healthy individuals (HC) and patients with osteoarthritis (OA). IL22 serum levels, measured by Enzyme-Linked Immunosorbant Assay, were highly elevated in RA patients compared with OA patients and HC. Comparably high levels of IL22 were detected in the synovial fluid of RA patients. Surprisingly, IL22 was only modestly elevated in the sera of PsA patients. Consistent with these findings, the cellular analysis revealed increased frequencies of IL22-producing cells in activated CD4 T cells from RA but not from PsA patients, as assessed by three-colour flow cytometry. The increased frequency of IL22 positive cells was primarily attributed to the enrichment of IL22/IL17 double-positive cells. As priming of CD4 $\mathrm{T}$ cells under optimal Th17 cell-inducing conditions in vitro resulted in an increase of IL22/IL17 double producing cells at the cost of IL17 single producers when compared with neutral priming conditions, the in vivo data from the patients suggest the presence of an environment facilitating biased Th17 cell development in RA but not in PsA.

Conclusion The data demonstrate increased IL22 expression and increased frequencies of IL17/IL22 double producing cells in RA. They therefore suggest the presence of an endogenous environment in RA promoting biased differentiation of inflammatory Th17 cells. 